\title{
Extended Capabilities of the Power Quality Management System of the Power Distribution Grid for Data Exchange
}

\author{
Denisa Rusinaru',*, Claudiu Popirlan ${ }^{1}$, Gabriel Stoian ${ }^{1}$, Cosmin Buzatu ${ }^{1}$, Andrei Negoita ${ }^{1}$, Leonardo Geo Manescu ${ }^{1}$, \\ Adrian Cojoaca $^{2}$ and Mihai Mircea ${ }^{1}$
}

${ }^{1}$ University of Craiova, INCESA (Research Hub for, Applied Sciences), 107 Decebal Bld, 200440 Craiova, Romania

${ }^{2}$ Oltenia Distribution, Craiova, Romania

\begin{abstract}
The actual operation of the power distribution systems asks for high power quality (PQ). This fully justifies the investments in improving the metrics of power grid performances. But maintaining a PQ data infrastructure in a numerous locations is time-consuming and prohibitive. Moreover, each PQ monitor releases its own data format. These considerations justified using of a central PQ management system able to manipulate elliptical and time discontinuous information. The paper presents the characteristics of a webbased application designed to collect the data measured with different technologies of monitors and translate them into a common PQ data interchange format allowing comprehensive and long-duration grid operation assessment. The unitary formatted data generated by this customized software tool can be further processed by a proprietary software platform for PQ management owned by the network operator. The present version of this conversion tool is applicable only for one product family operating in the local power distribution grid. Further development is planned for integrating other two monitor vendors/families.
\end{abstract}

\section{Introduction}

The nature of the actual power quality (PQ) issues fully justifies the investments for improving the metrics of the power grid operator performances as a key measure in increasing: the reliability, efficiency, as well as the satisfaction of the power grid's users. In accordance with the national Performance Standard for Power Distribution Service [1], the power distribution grids' operator (DGO) must assume the obligations regarding keeping within standards of the power reliability and quality.

The performance assessment and reporting operation, as well as the understanding of the operational problems, is improved by processing high-precision and comprehensive measurement data. Consequently, a utility or customer can be tempted to monitor more points than they can justify financially. Maintaining a PQ data collection infrastructure in a very large number of locations over a very long time is time-consuming and prohibitive (the cost of an equipment with required performance amounts to around 10000 Euros). Moreover, this leads the power utilities to cope with GB's of real data. This data "avalanche" is supplied by a diversity of equipment: monitors, analyzers, loggers, power meters, derived from various vendors and technologies. So that, the management of PQ database becomes critical $[2,3]$ and the high amount of collected data will ask for advanced analysis tools. This greatly increases the cost and difficulty of managing the PQ.

Moreover, most of the monitoring equipments have own software that can be used as platforms for database management. The development and use of a standard power quality data interchange format will make this data accessible for analysis, so that the utility or customer can increase the number of monitored points without large capital outlays for equipment. The standard exchange format will also enable the user to change equipment vendors without concern about incompatibilities with existing equipment. The data exchange format as COMTRADE (Common format for Transient Data Exchange for power systems) or PQDIF (Power Quality Data Interchange Format IEEE ${ }^{\circledR}$ Std 1159.3-2003 standard) can provide vendors with a common format for the exchange of data, allowing the end user maximum flexibility in choice of tool and vendor $[4,5]$.

Therefore, specialized analysis can be performed with an integrated platform as PQView ${ }^{\circledR}$ (PQ management and analysis software system) [6]. Such kind of system is currently used by the local DGO. An extension of its functionality is a project (SYMMPQI) in which the local DGO is currently involved in a partnership with INCESA Research Hub for Applied Sciences (University of Craiova).

The paper presents the characteristics and applications of one of the SYMMPQI project's outputs [7]. SYMMPQI_PQDM is a customized software module that basically collects the output data measured with different technologies of PQ monitors and translate them into a unitary format - PQDIF standard. With clear procedures for data acquisition and storage, as well as methods of these data rebuilding and administration,

\footnotetext{
* Corresponding author: denisa.rusinaru@incesa.ro
} 
these data in PQDIF format can be unitarily processed by the platform PQView.

The present development of SYMMPQI_PQDM web-based application is applicable only for one class family product operating in the local DGO installation. Further development of the application is intending to address to other equipment technologies.

\section{National regulation framework for $P Q$ in the power distribution grids}

Presently, a considerable number of European countries use the European standard EN 50160 as the basis for their national quality of power supply regulations. According to EN 50160 ("Voltage characteristics of electricity supplied by public distribution systems") [8], accommodated by the Romanian Performance Standard for Power Distribution Service (PSPDS), the recommended levels of different PQ parameters are specified in the time-based percentage.

According to PSPDS, four PQ disturbances should be guaranteed by DGO at the interface with its grids users: slow voltage variations, voltage fluctuations, rapid voltage changes (normal operation), unbalances voltage, and waveform distortions. PSPDS considers the following objectives for PQ parameters:

1) Slow voltage variations

During one week, under normal operating conditions: (1) $95 \%$ of the 10 -min RMS voltage should be within $U_{n}$ $\pm 10 \%$ and $100 \%$ of the 10 -min RMS voltage should be within $+10 \% /-15 \%$ for LV system; (2) $99 \%$ of the 10 min RMS voltage should be within $\mathrm{U}_{\mathrm{c}} \pm 10 \%$ and $100 \%$ of the 10-min RMS voltage should be within Uc $\pm 15 \%$ for MV and $\mathrm{HV}$ system, where $\mathrm{U}_{\mathrm{n}}$ is the nominal voltage of the system and $U_{c}$ is the declared supply voltage.

2) Voltage fluctuations

The long-term flicker severity, $\mathrm{P}_{\mathrm{It}}$ is calculated from a sequence of $12 \mathrm{P}_{\mathrm{st}}$ values (short-term 10 min flicker severity) over a 2 -hours interval, according to (1):

$$
P_{l t}=\sqrt[3]{\frac{\sum_{1}^{12} P_{s t, i}^{3}}{12}}
$$

The 95th percentile value of $P_{1 t}$ should not exceed the unity over one week.

3) Supply voltage unbalance

According to IEC definition, the negative sequence voltage unbalance factor is:

$$
V U F=\frac{V_{2}}{V_{1}} \cdot 100=\sqrt{\frac{1-\sqrt{3-6 \beta}}{1+\sqrt{3-6 \beta}}} \cdot 100
$$

where $\beta=\frac{V_{a b}^{4}+V_{b c}^{4}+V_{c a}^{4}}{\left(V_{a b}^{2}+V_{b c}^{2}+V_{c a}^{2}\right)^{2}}$

with $V_{\mathrm{ij}}$ - the RMS line-to-line voltage

$\mathrm{V}_{2}$ - negative sequence voltage

$\mathrm{V}_{1}$ - positive sequence voltage.

For LV and MV systems, PSPDS regulates the $95 \%$ probability weekly value of negative-sequence voltage unbalance factor within $2 \%$, with $1 \%$ in HV systems.

4) Harmonic distortion

In accordance with PSPDS, the $95 \%$ value of the total voltage harmonic distortion factor THD (defined as in (3)) shall not exceed $8 \%$ for the LV and MV system in any period of a week, under normal operating conditions. In the case of $\mathrm{HV}$ grid, the objective is $3 \%$.

$$
T H D=\frac{\sqrt{\sum_{2} V_{h}^{2}}}{V_{1}} 100
$$

where $\mathrm{V}_{\mathrm{h}}$ is the RMS value of the $h$-th harmonic component; $\mathrm{V}_{1}$ - the RMS value of the fundamental component.

The DGO's are obliged to submit to the Romanian Energy Regulatory Authority the detailed annual PQ assessment report for their operated networks, which includes states of compliance with the PQ limits referred to the PSPDS and in accordance with Std. EN 50160.

\section{Power Quality monitoring systems}

In the Romanian power networks, there are voltage quality monitoring systems at both transmission and distribution level. The transmission and distribution operators are responsible for the network voltage waveform quality. They have the duty to look out for the levels of each characteristic.

Following this trend, the local DGO has placed PQ recorders presently organized within three fixed monitoring systems: (1) the monitoring system for 110 $\mathrm{kV}$ and $20 \mathrm{kV}$ grids (i.e. equipped with monitors MAVOSYS for almost 100 sites) connected to a central server and managed by PQView multi-component system; (2) the monitoring system at the interface between the distribution network and its users, without a data management center; (3) the power monitors system (i.e. MEG) connected on the LV side of $20 / 0.4 \mathrm{kV}$ substations.

Usually, each PQ monitor produces its own data file format. The DGO decision regarding one or other type of meter is associated with interoperation ability and related standards. These considerations have justified the using of a central PQ management system in compliance with the National Performance Standard for Power Distribution Service (PSPDS) and able to manipulate rather an elliptical and time discontinuous information for assessing the network's observability.

Since the PQView accepts formats from large categories/types of PQ monitors, it looks like a convincing solution to the need of DGO for transferring the output of different vendors' monitors and simulation programs to a common database and analysis program. Mainly it is using a standard data interchange format, e.g. PQDIF. For the case of other vendors' equipment, PQView should be assisted by a data handler for these specific formats.

In the present configuration of the PQ system of the local DGO only $22.4 \%$ of the permanent monitoring equipment can provide PQ data in PQDIF format. The rest of them export data in proprietary formats, as the case of the MEG analyzers placed in LV buses of the network - see Fig.1. Though less than $14 \%$ of the equipments (MAVOSYS type) are currently integrated with the PQView software platform, the perspective is in the favor of a major inclusion. Additional tools, as SYMMPQI_PQDM are developed for PQDIF 
conversion of the data achieved from MEG equipmentbased monitoring system.

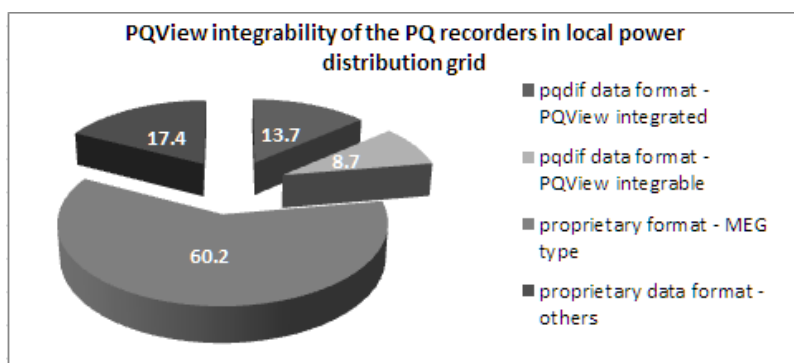

Fig. 1. PQView integrability stage of the PQ monitors in the local power distribution grid

\section{Data handling for $P Q$ analysis}

By using a centralized system for the PQ management and analysis in its networks, the DGO benefits of the evaluation of all of the PQ parameters for individual sites and their zonal global assessment; establishing references for the system's expected performances regarding all the PQ categories; integrated technical-economic evaluation of the PQ problems and their solutions; web-based access to the PQ information; useful tool for training in the PQ field.

The data asked for analysis is provided by the socalled Points of Interest System (PoI). Actually, these are the metering system components of DGO including a high number of heterogeneous families, types and classes of equipment: A or S class, PQ monitors, digital fault recorders, microprocessor relays, SCADA systems, demand and energy meters, operating logs. These devices differ in their data format and the method of measured quantities aggregation. In the case of the general power/energy meters, the data are accessed as *.txt, *.xls or *.csv files. In the case of the PQ monitoring, the data should be preferable accessible in standard formats as PQDIF, which enables the data exchange between software applications.

For this purpose, the PQView integrates data from PQ monitors into an open relational database, but presently only for the HV \& MV grid area. At the LV level, the PQ data are collected either on-site or remotely from a MEG40-based monitoring system, being uploaded and stored on a dedicated resource server. Originally, the MEG family equipments use own proprietary communication protocols, as well as data storage and interpretation modes for PQ monitoring.

The present stage of interfacing the PQ monitoring system of the local DGO with the PQView's database is given in Fig. 2.

Since the PQView system can ensure the full availability of data if only these are retrieved in pqdif formats, a translation of other proprietary data format should be accomplished if a PQView integration is desired. A solution is supplied by converting these data into an easy-to-handle format, e.g. csv. This format enables further conversion in PQDIF standard, making possible the unification of measurements from different DGO family equipments: MAVOSYS, MEG in the present, and ION, Chauvin Arnoux, Fluke in next stage.

The principle of this approach is using the existing software to extract data under a commonly accepted "csv" format (where applicable), followed by converting "csv" files to "pqdif" files compatible with the existing PQView software.

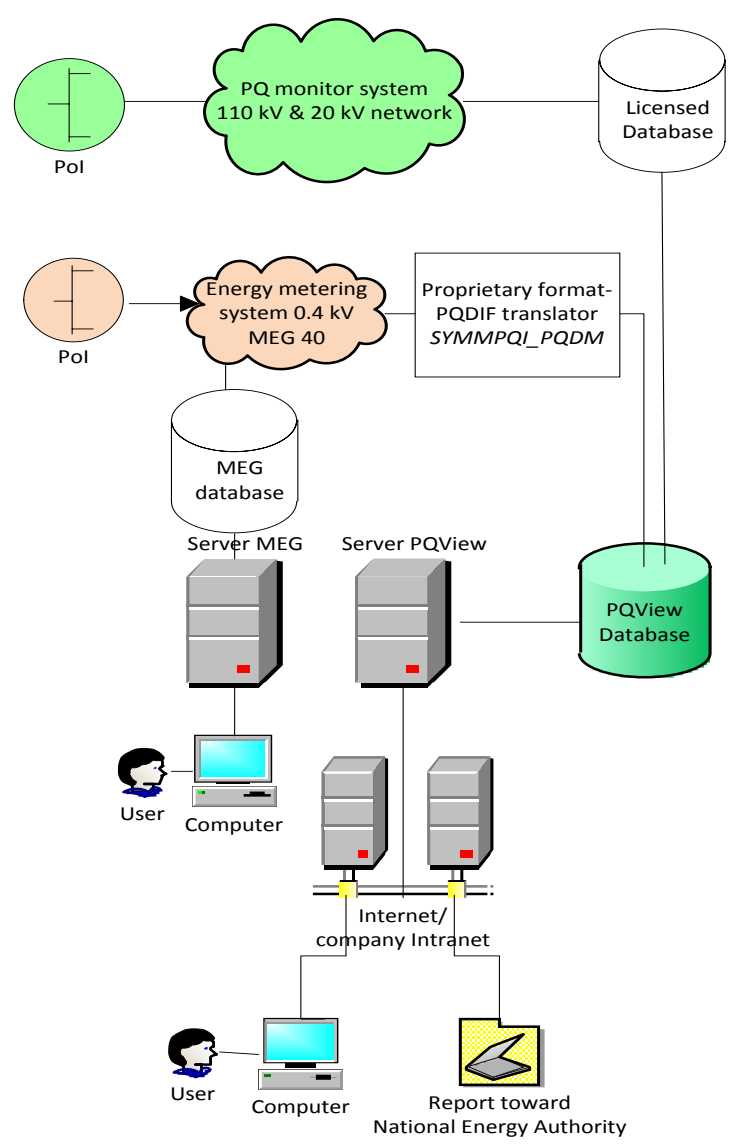

Fig. 2. Schematic of the solution of $P Q$ monitor interfacing with PQView system of the local DGO

SYMMPQI_PQDM is a customized tool that allows using and extending the capabilities of the PQView platform. SYMMPQI_PQDM is organized on modules with functions for managing both the monitoring of data and the configuration of the data transfer tools.

Table 1. Functions of the modular structure of SYMMPQI

\begin{tabular}{|c|c|c|}
\hline \multirow{2}{*}{ SYMMPQI } & $\begin{array}{c}\text { Data collection } \\
\text { function }\end{array}$ & Energy measurement \\
\cline { 2 - 3 } & \multicolumn{2}{|c|}{ Tools'parametrization function } \\
\cline { 2 - 3 } & \multicolumn{2}{|c|}{ PQDIF translator function } \\
\cline { 2 - 3 }
\end{tabular}

SYMMPQI_PQDM includes two modules: Power Quality Data Management Web Application (PQDM Web) and Power Quality Data Collection (PQDC), as it can be seen in Fig. 3 [7].

In the current version, the PQDM Web tool ensures a deterministic way to get and collect measurements from the MEG-system, either manually or automatically. PQDM Web performs further the conversion of raw data collected in PoI of MEG40 type ("csv" format) to PQDIF. The most convenient way of data acquisition is 
the access to the analyzer database by means of SQL query. The data is further prepared for the next step: uploading into the PQ analysis and report system (PQView Data Analyzer).

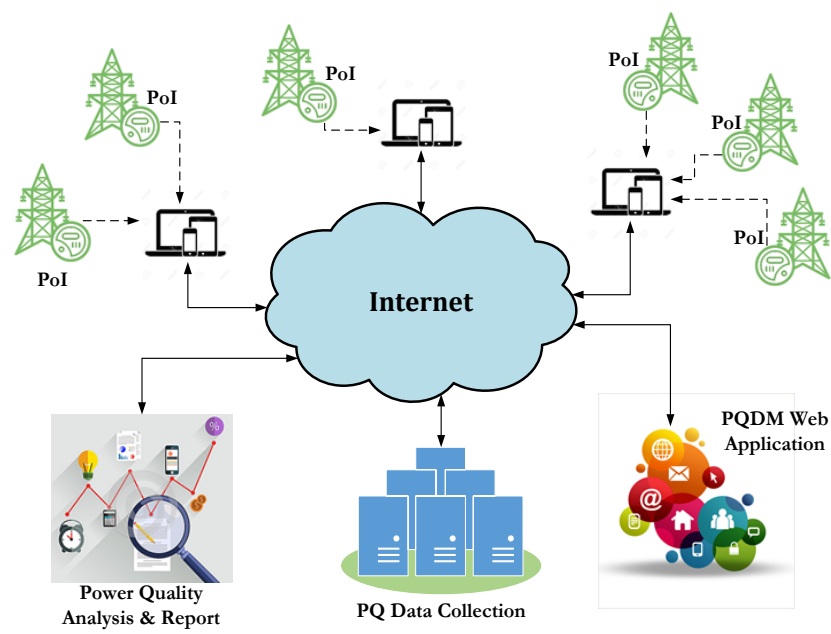

Fig. 3. SYMMPQI-PQDM Architecture [7]

The main functions of PQDM Web module are: - Reading the metering data in the native format ( $m$ dat for MEG) and conversion into intermediary format (csv);

- Analysis of the data consistency;

- Data conditioning by recovering error/missing data;

- Building intermediary database;

- Data conversion into user proprietary format pqdif).

PQDM Web application is designed to support any type of acquisition equipment by integrating an appropriate plugin able to process its information. In this way, our system is easy to be expanded and maintained. The PQDM-WebApp application is developed using Java technologies, in NetBeans development environment (IDE). It is running in the Web environment. It has the advantages of exploiting Oracle J2EE which brings robustness, independence from the platform, and efficiency, as well as possibility of using various application servers (case of Apache Tomcat).

PQDM Web Application stores its outputs in a PQDC (Power Quality Data Collection) site. PQDC can be collocated with the web application or can be distributed. Smaller organizations can opt to the collocated solution, while bigger ones can choose the distributed variant. DGO is going to decide about the version, in accordance with its available IT resources. Either of solutions, it provides a unique place from where all PQ information can be retrieved by software packages used for various analyses and reports.

The resulted "pqdif" data files are operated by the PQView Data Analyzer module (PQDA). PQDA contains functions performed by the Excel spreadsheet and the MS Access database. The PQ data can be also stored in the database of PQView Data Manager.

\subsection{Data extraction and collection}

PQ monitors are delivered in a fixed built-in version.

Data upload can be achieved by direct communication (USB/SERIAL RS232) or Ethernet communication protocol from the measuring devices using the software either provided by manufacturers of measuring instruments or as module of existing measurement systems.

The data taken from the measuring devices is stored in the primary format - " $c s v$ " - by means of either:

a manufacturer's dedicated software: e.g. Meg4X, MegaMerci or DataViewer for MEG40 (located in LV power grid), DranView or PQDiffractor for Mavosys10 (located in HV \& MV power grid);

- a customized software module: e.g. SYMMPQI_PQDM applicable presently only for MEG40 devices.

Further, the data are converted to "pqdif" format for advanced storage, processing and analysis within the PQView application.

\subsection{Collection of data from MEG40-based monitoring system}

The retrieving and processing data from MEG40 devices can be achieved by using the proprietary program package MEgA_Merci_II Originally, the measured values of the defined quantities can be stored as MDAT format files for $\mathrm{MEG}^{*}$ 's. The file created in this way can be further used for the WebDator system or for DVMEg.exe (an MDAT file viewer) [9].

The output data are stored on a dedicate server, allowing to DGO to perform a basic and single-site analysis of the voltage evolution and power/energy consumption in the monitored grid area.

In the same way, the customized software module, SYMMPQI_PQDM, can be used to retrieve and process data from MEG40 devices. Basically, PQDM-Web application receives the *.csv file for the MEG40 together with the user input (Version Info, File Name, Creation, Subject, Author, Application, Copyright, Trademark, Notes, Language, Owner) and returns the corresponding *.pqdif file. The measurement data flow for format conversion by SYMMPQI_PQDM and PQView integration is given in Fig. 4.

In order to evaluate the *.pqdif files generated by each MEG monitor, it is necessary to subject the monitors to an assortment of known voltage and current variations. In this way, the resulting PQDIF file can be compared to the known disturbance and the data recorded by the monitor.

In order to test the functionality of SYMMPQI_PQDM translator, several preparatory operations were required:

1. Establishing the used communication protocol (MODBUS), the necessary communication parameters and the commands to retrieve the data from the memory card of the measuring device.

2. Establishing the data storage mode - the storage is in ASCII code, with some features in the way of marking the measurements and calculating the parameters.

An example of identifying measurements and calculating a parameter such as voltage is given in Fig. 5, in hexadecimal form, with the correspondent resulting *.csv file given as in Fig. 6 . 
Further, in order to browse, test and diagnose the converted PQDIF files, three electrical quantities evolutions recorded by MEG40 as PoI located in a LV bus with PV power plant interconnected were followed.

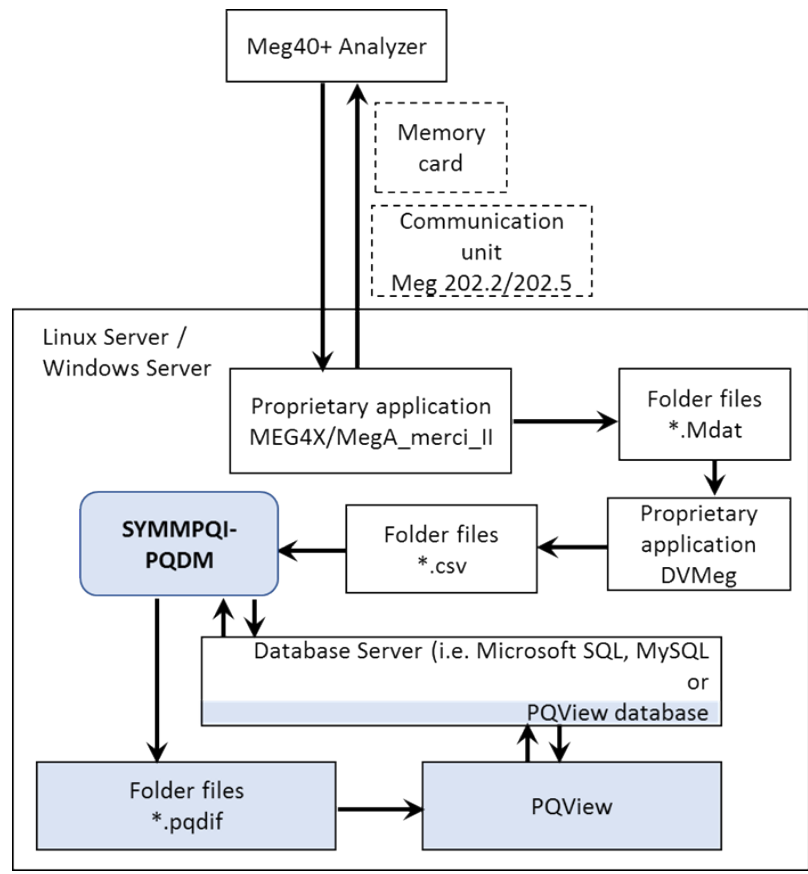

Fig. 4. Data flow between LV analysers and PQView platform

The correspondence between graphical evolution plotted in .csv original report of the MEG monitor and plot of variation in PQView-PQDA graphical report is verified.

$2 \mathrm{~d} b 8$ Ob oe $2 \mathrm{dCR}$ CR

$4 a \cdot 2 c \cdot 55 \cdot 2 d \cdot 1 c \cdot 2 e \cdot 0 a \cdot 2 d \cdot 49 \cdot 2 c \cdot 54 \cdot 2 d \cdot 16 \cdot 2 e \cdot 03 \cdot 2 d \cdot 44 \cdot 2 c \cdot 4 f \cdot 2 d \cdot 12 \cdot 2 e \cdot 09 \cdot 00 \cdot 09 \cdot 00 \cdot 09 \cdot 00 \cdot 09 \cdot 00 \cdot 00 \cdot 00$ CRR

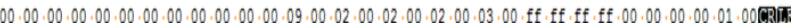

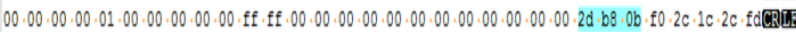

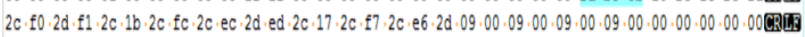

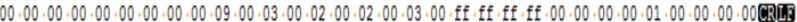

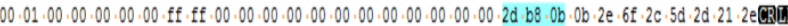
$06 \cdot 2 \mathrm{e} \cdot 6 \mathrm{c} \cdot 2 \mathrm{c} \cdot 5 \mathrm{sb} \cdot 2 \mathrm{~d} \cdot 1 \mathrm{~d} \cdot 2 \mathrm{e} \cdot 00 \cdot 2 \mathrm{e} \cdot 67 \cdot 2 \mathrm{c} \cdot 57 \cdot 2 \mathrm{~d} \cdot 18 \cdot 2 \mathrm{e} \cdot 09 \cdot 00 \cdot 00 \cdot 00 \cdot 08 \cdot 00 \cdot 09 \cdot 00 \cdot 00 \cdot 00 \cdot 00 \cdot 00 \cdot 00 \cdot 00 \cdot 00 \cdot 00 \mathrm{CR}$. $00 \cdot 00 \cdot 00 \cdot 00 \cdot 00 \cdot 00 \cdot 09 \cdot 00 \cdot 02 \cdot 00 \cdot 02 \cdot 00 \cdot 02 \cdot 00 \cdot 03 \cdot 00 \cdot$. ff eff eff eff $\cdot 00 \cdot 00 \cdot 00 \cdot 00 \cdot 01 \cdot 00 \cdot 00 \cdot 00 \cdot 00 \cdot 00 \cdot 01 \cdot 00$ CRR

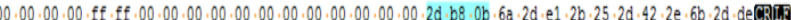

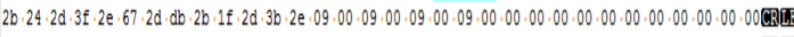
$00 \cdot 00 \cdot 00 \cdot 09 \cdot 00 \cdot 03 \cdot 00 \cdot 02 \cdot 00 \cdot 02 \cdot 00 \cdot 03 \cdot 00$. ff ff ff ff ff ff ff $00 \cdot 00 \cdot 01 \cdot 00 \cdot 00 \cdot 00 \cdot 00 \cdot 00 \cdot 01 \cdot 00 \cdot 00 \cdot 00 \cdot 00$ CRER

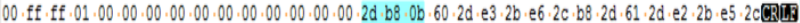

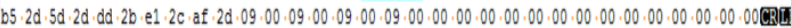

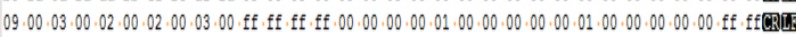

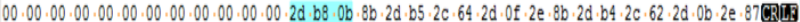

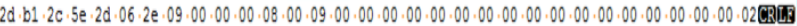

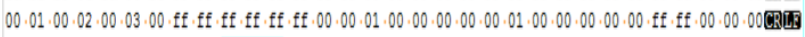
$00 \cdot 00 \cdot 00 \cdot 00 \cdot 00 \cdot 00 \cdot 00 \cdot 00 \cdot 00 \cdot 2 \mathrm{~d}$ b8 $0 \mathrm{~b} \cdot 80 \cdot 2 \mathrm{c} \cdot 93 \cdot 2 \mathrm{~b} \cdot 0 \mathrm{a} \cdot 2 \mathrm{~d} \cdot 96 \cdot 2 \mathrm{~d} \cdot 7 \mathrm{~d} \cdot 2 \mathrm{c} \cdot 8 \mathrm{e} \cdot 2 \mathrm{~b} \cdot 09 \cdot 2 \mathrm{~d} \cdot 95 \cdot 2 \mathrm{~d} \cdot 79 \cdot 2 \mathrm{c} \cdot 89 \cdot 2 \mathrm{bCR}$ C. $05 \cdot 2 \mathrm{~d} \cdot 91 \cdot 2 \mathrm{~d} \cdot 09 \cdot 00 \cdot 09 \cdot 00 \cdot 09 \cdot 00 \cdot 09 \cdot 00 \cdot 00 \cdot 00 \cdot 00 \cdot 00 \cdot 00 \cdot 00 \cdot 00 \cdot 00 \cdot 00 \cdot 00 \cdot 00 \cdot 00 \cdot 00 \cdot 00 \cdot 00 \cdot 00 \cdot 02 \cdot 00 \cdot 02 \cdot 00 \mathrm{CR}$.

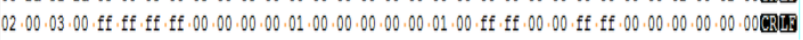
$00 \cdot 00 \cdot 00 \cdot 00 \cdot 00 \cdot 00$

Identification of measurements data in raw format

\begin{tabular}{|l|l|l|l|}
\hline & & DEC & Rezultat : \\
\hline ADC-HEX & Ob & 11 & \\
\hline Offset-HEX & $2 \mathrm{e}$ & 46 & \\
\hline Rezolutie & 0.05 & & \\
\hline Referinta Offset & 5 & & \\
\hline Calculat & & & \\
\hline
\end{tabular}

Calculation of the medium voltage on A-phase.

Fig. 5. Preparatory operation for PQDM retrieving identifying/converting of raw measurement from MEG40

The steps for testing the functionality of SYMMPQI_PQDM translator are given in Fig. 7-9.

The "csv" file of output data (graphical correspondence given in Fig. 7-9 (a)) are proccesed and concerverted as "pqdif" format, and further uploaded by PQView - PQDA module (graphical report correspondnce given in Fig. 7-9 (c)). The consistency of the new generated "pqdif" files is preliminary tested/validated by using the viewer PQDiffractor (a free PQDIF file viewer utility written by Electrotek Concepts) [10] - see Fig. 7-9 (b).

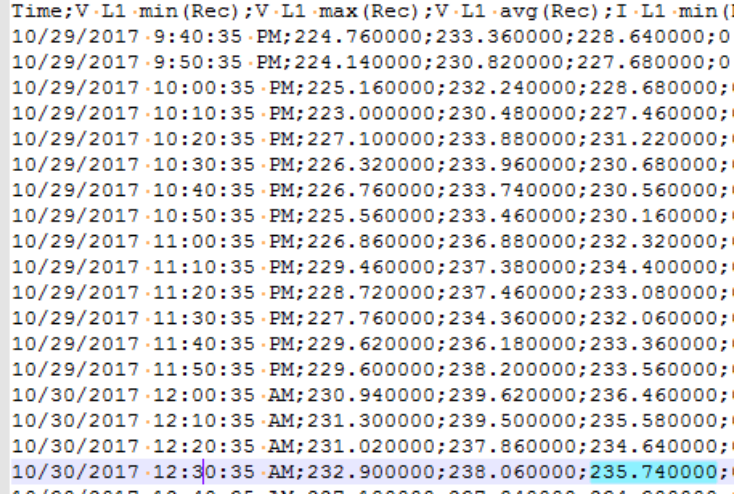

10/30/2017 -12:310:35 AM;232.900000;238.060000;235.740000;

Fig. 6. Resulting *.csv file after processing
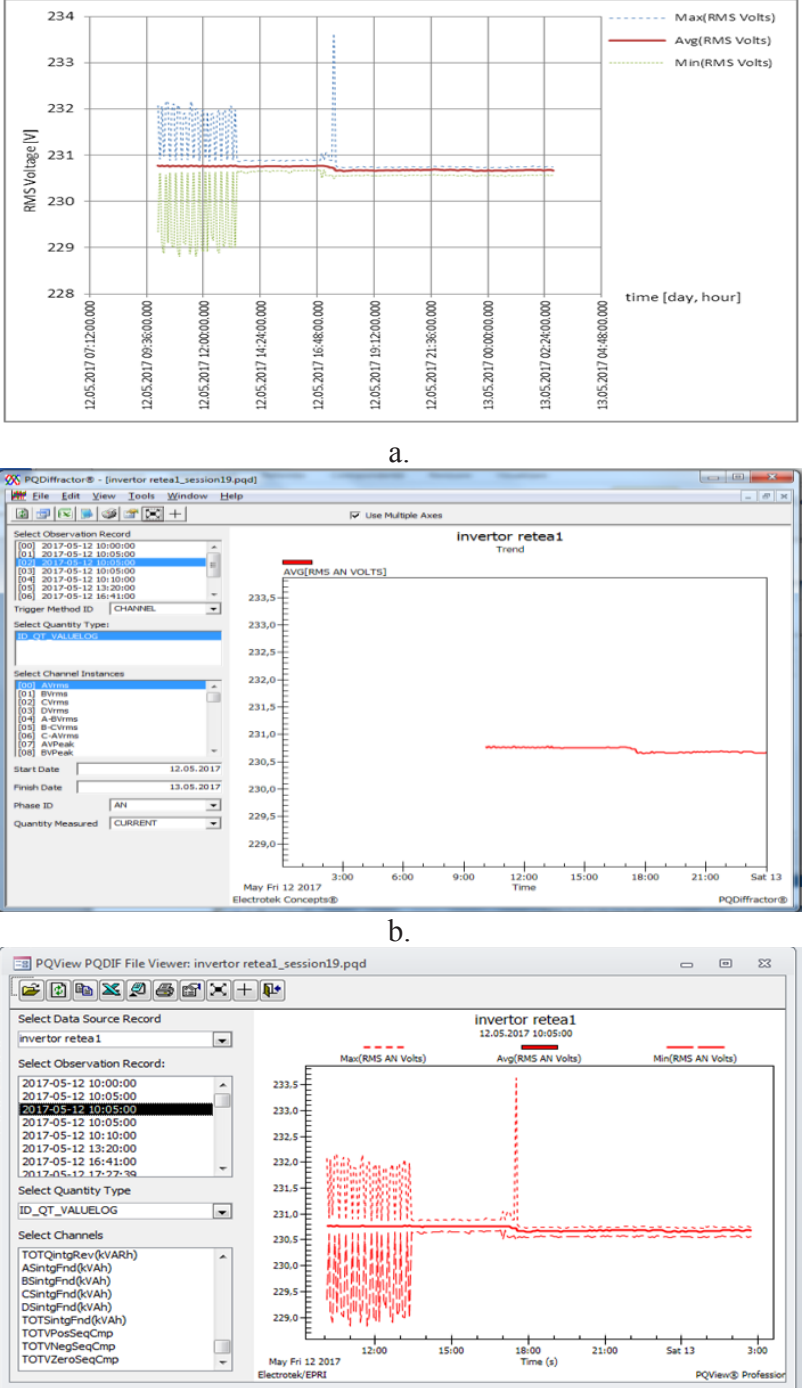

Fig. 7. RMS voltage variation on the surveilled site: a. graphical output of primary csv file of MEG; b. pqdif file consistency check using PQDiffractor; c. PQView IEEE PQDIF File viewer processing 


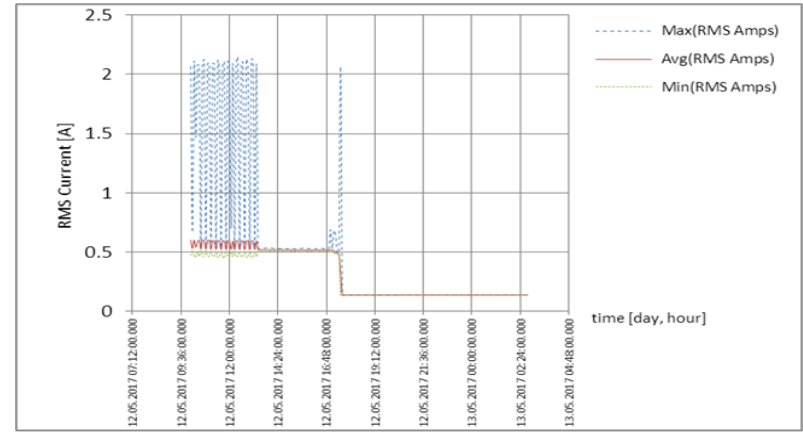

a.

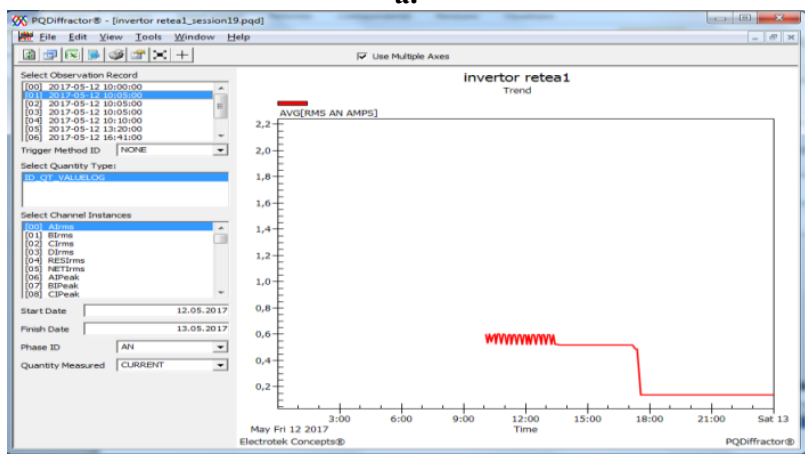

b.

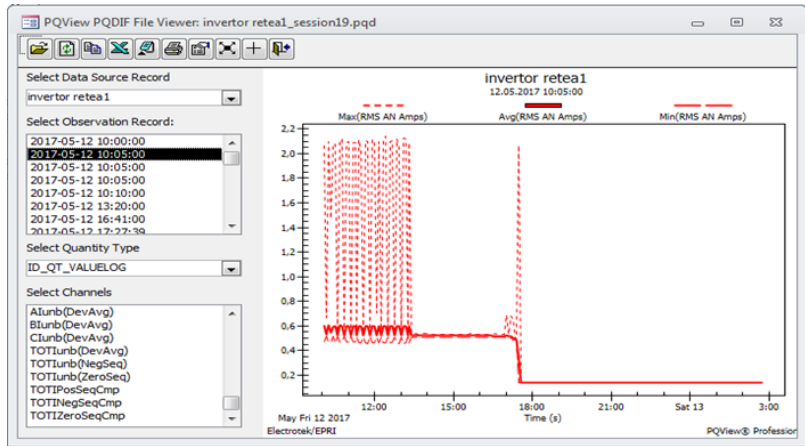

c.

Fig. 8. RMS current variation on the surveilled site: a. graphical output of primary csv file of MEG; b. pqdif file consistency check using PQDiffractor; c. PQView IEEE PQDIF File viewer processing

\subsection{Report content of PQView integrated recorded data}

Based on continuous monitoring, platform PQView is able to summarize variations of electrical values and PQ parameters with time trends and statistics, in addition to characterizing individual events.

The following data are usually provided based on the measurements performed by the PQ monitors integrated or integrable in PQView platform - see Table 2.

Based on these data, the analysis module of PQView, PQDA allows creating reports including the trends, histograms, and statistics for any steady-state voltage, current, power, and energy quantity.

Because the EN50160 Std, as well as PSPDS is based on $95 \%$ values, the reporting module of the PQ software should calculate them. These reports can automatically summarize the PQ monitoring campaigns or the compliance with EN 50160 at the all voltage levels of the power distribution grid.

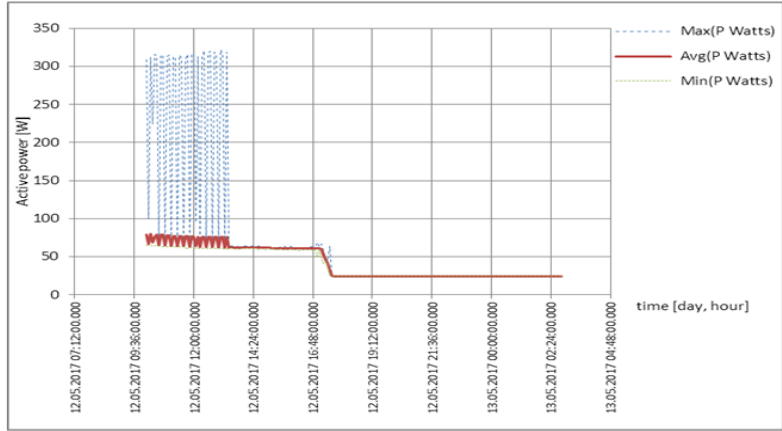

a.

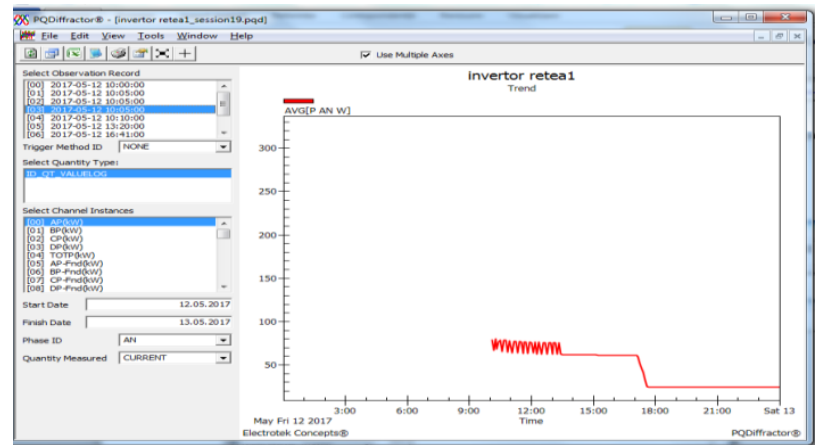

b.

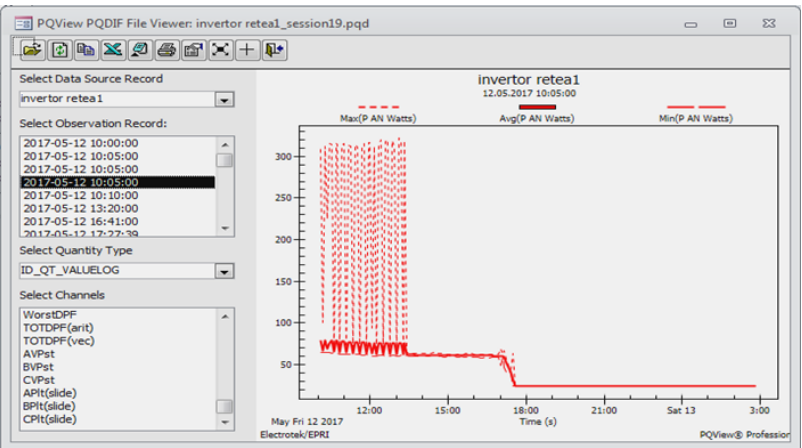

c.

Fig. 9. Active power variation on the surveilled site: a. graphical output of primary csv file of MEG; b. pqdif file consistency check using PQDiffractor; c. PQView IEEE PQDIF File viewer processing

Table 2. Report of measured data by PQ monitors in power distribution grid

\begin{tabular}{|c|c|c|c|}
\hline $\begin{array}{l}\text { Text \& } \\
\text { graphical } \\
\text { information on } \\
\text { PQ } \\
\text { parameters }\end{array}$ & $\begin{array}{l}\text { MEG40 } \\
\text {-separate } \\
\text { LV } \\
\text { system }\end{array}$ & $\begin{array}{l}\text { MEG - } \\
\text { PQView } \\
\text { integrated (by } \\
\text { SYMMPQI_- } \\
\text { PQDM) } \\
\end{array}$ & $\begin{array}{l}\text { MAVOSYS } \\
-\quad \text { PQView } \\
\text { integrated }\end{array}$ \\
\hline Voltage values & $\mathrm{X}$ & $\mathrm{X}$ & $\mathrm{X}$ \\
\hline $\begin{array}{l}\text { Current values } \\
\text { (min, avg, max) }\end{array}$ & X & $\mathrm{X}$ & X \\
\hline $\begin{array}{l}\text { Power values } \\
\text { (min, avg, max) }\end{array}$ & X & $\mathrm{X}$ & $\mathrm{X}$ \\
\hline Energy & X & $\mathrm{X}$ & X \\
\hline $\begin{array}{l}\text { No of transitory } \\
\text { interruptions }\end{array}$ & X & $\mathrm{X}$ & $\mathrm{X}$ \\
\hline $\begin{array}{l}\text { No of short } \\
\text { interruptions }\end{array}$ & $\mathrm{X}$ & $\mathrm{X}$ & $\mathrm{X}$ \\
\hline $\begin{array}{l}\text { No of long } \\
\text { interruptions }\end{array}$ & X & $\mathrm{X}$ & $\mathrm{X}$ \\
\hline $\begin{array}{l}\text { No of voltage sags } \\
\text { Medium }\end{array}$ & $\mathrm{X}$ & $\mathrm{X}$ & $\mathrm{X}$ \\
\hline frequency $(\mathrm{Hz})$ & X & $\mathrm{X}$ & $\mathrm{X}$ \\
\hline
\end{tabular}




\begin{tabular}{|c|c|c|c|}
\hline $\begin{array}{l}\text { Text \& } \\
\text { graphical } \\
\text { information on } \\
\text { PQ } \\
\text { parameters }\end{array}$ & $\begin{array}{l}\text { MEG40 } \\
\text {-separate } \\
\text { LV } \\
\text { system }\end{array}$ & $\begin{array}{l}\text { MEG - } \\
\text { PQView } \\
\text { integrated (by } \\
\text { SYMMPQI_ } \\
\text { PQDM) }\end{array}$ & $\begin{array}{l}\text { MAVOSYS } \\
-\quad \text { PQView } \\
\text { integrated }\end{array}$ \\
\hline $\begin{array}{l}\text { RMS voltage } \\
\text { variations } \\
\text { (value \%, } \\
\text { weeks number) }\end{array}$ & - & $X$ & $X$ \\
\hline $\begin{array}{l}\text { Limit violations } \\
\text { for rapid } \\
\text { voltage } \\
\text { variations } \\
\text { (no/year) }\end{array}$ & - & - & $X$ \\
\hline $\begin{array}{l}\text { Temporary } \\
\text { phase-to- } \\
\text { ground }\end{array}$ & - & - & $X$ \\
\hline $\begin{array}{l}\text { overvoltage at } \\
50 \mathrm{~Hz} \% \text { value } \\
\text { / week })\end{array}$ & - & - & $X$ \\
\hline $\begin{array}{l}\text { Limit violations } \\
\text { for long term } \\
\text { flicker } \\
\text { (no/week) }\end{array}$ & - & - & $X$ \\
\hline $\begin{array}{l}\text { Limit violations } \\
\text { for h-th voltage } \\
\text { harmonic (\% of } \\
\text { fundamental } \\
\text { week) }\end{array}$ & - & - & $\mathrm{X}$ \\
\hline $\begin{array}{l}\text { Limit violations } \\
\text { for THD ( } \% \\
\text { value/week) }\end{array}$ & - & - & $X$ \\
\hline $\begin{array}{l}\text { Limit violations } \\
\text { for voltage } \\
\text { unbalanced } \\
\text { factor } \\
\text { value/week) }\end{array}$ & - & $X$ & $X$ \\
\hline $\begin{array}{l}\text { Cumulative } \\
\text { frequency } \\
\text { histogram }\end{array}$ & - & $X$ & $X$ \\
\hline
\end{tabular}

Table 2. Reporting of measured data for PQ monitors in power distribution grid (continuation)

The original information can be managed in PQDIF data file format within the PQView database.

\section{Conclusions}

Comprehensive information regarding the PQ level at the interconnection buses between the power distribution grids and their users allows a better assessment of the system operational conditions, the causes of PQ disturbances and the appropriate mitigation measures.

Therefore, the local DGO has registered important efforts for developing a high-performance system for permanent $\mathrm{PQ}$ monitoring in its grids, no matter the voltage level. A complex software application PQView was implemented in order to manage and analyze the PQ data supplied by this system. The PQView database is presently used by DGO according to a standard monitoring procedure for setting the global or local PQ level in its networks.

Some other extended functions are further added to the existent PQ management system in order to facilitate the unitary analysis of heterogeneous measurement data provided by different families/vendors equipments. An in-house developed web-based application was developed in the framework of a knowledge transfer project between power distribution company and university. The main function of the SYMMPQI_PQDM is retrieving and processing the data provided by a certain category of LV monitors equipment, by converting their native format of the output data into a PQDIF Standard, for further integration into the central platform for the PQ management and analysis in the power distribution grids.

The results of the checking procedure regarding the actual stage implementation of this tool are presented.

\section{Acknowledgements}

This work was supported by a grant of the Romanian National Authority for Scientific Research and Innovation, CNCS/CCCDI - UEFISCDI, project number PN-III-P2-2.1BG-2016-0269, within PNCDI III.

\section{References}

1. *** Romanian Standard The Electricity Distribution Service - Standard of Performance (OG 11/2016), www.anre.ro (2016)

2. M. Music, A. Bosovic, N. Hasanspahic, S. Avdakovic, E. Becirovic, Integrated Power Quality Monitoring Systems in Smart Distribution Grids, 2012 IEEE International Energy Conference and Exhibition (ENERGYCON), (2012)

3. A. Mubiana, M. Mutingi, G.Gope, C. Mbohwa, Analysis of Power Quality Factors: A Case Study of Windhoek, Namibia, Proceedings of the World Congress on Engineering and Computer Science 2016 Vol II WCECS 2016, October 19-21, 2016, San Francisco, USA (2016)

4. *** IEEE Std 1159.3 Recommended Practice for the Transfer of Power Quality Data, IEEE, (2008)

5. Power Quality Data Interchange Format (PQDIF) Software Verification and Demonstration Test Bed for Power Quality and Advanced Revenue Meters

6. Electrotek Concepts, Inc., PQView - User Manual and Quick Start Guide, www.pqview.com

7. L.G. Manescu, D. Rusinaru, C. Popirlan, G. Stoian, M. Ciontu, C. Buzatu, a.o., Complex Software System for Data Management and Analysis of Power Distribution Grids, 4th International Conf. on Mathematics and Computers in Sciences and Industry MCSI 2017, Corfu, Greece, (2017)

8. *** EN 50160, Voltage characteristics of electricity supplied by public distribution systems, (2010)

9. PQ monitors - voltage quality, avalable at http://www.e-mega.cz/meg-40

10. PQDiffractor 2.7.3 Released, available at http://www.pqview.com/new/2016/6/17/pqdiffractor -273-released.html 